\title{
An Acupuncture Prescription Suggested for Treating Primary Dysmenorrhea
}

\author{
Tong Zheng Hong* \\ As-You-Wish Healthcare Institute, Taiwan
}

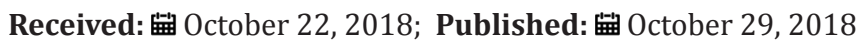

*Corresponding author: Tong Zheng Hong, As-You-Wish Healthcare Institute, MS in Acupuncture awarded by National University of Health Sciences in IL, Taiwan

\begin{abstract}
Primary dysmenorrhea (PM) is the issue that needs attention because most of the women during reproductive age are affected with it, causing discomfort in daily life. Acupuncture has been seen as an alternative therapy and been considered "essential health benefits" in the western countries with more and more popularity, which has confirmed TCM and acupuncture effective as Western medications for PM. However, all the TCM and acupuncture must follow pattern identification for the best results.
\end{abstract}

Keywords: Primary dysmenorrhea; NSAIDs; pattern identification

\section{Introduction}

It is estimated the percentage of women affected by dysmenorrhea ranges from $16 \%$ to $90 \%$. Dysmenorrhea, the most prevalent gynecological issue with the women during reproductive age, refers to the occurrence of painful uterine cramping associated with menses, which occurs before, during and/or after menstrual periods with symptoms, such as low pelvic discomfort/pain, low back pain, pain radiated to the anterior thighs, nausea, vomiting, diarrhea, headache, sacral pain, starting 1 to 3 days before the onset of menses [1]. In general dysmenorrhea is classified to Primary Dysmenorrhea (PD), which refers to pain that is not associated with obvious pelvic disease and typically begins with the onset of ovulatory cycles right after menarche. It has been reported the prevalence rate of primary dysmenorrhea is as high as $90 \%$. On the other hand, Secondary Dysmenorrhea (SD) closely related to cervical stenosis, endometriosis and typically has a later onset, usually develops in women who have previously had normal periods [2]. Clinically menstrual pain counts for $37 \%$ of the menstrual dates on average, and ranges from $56.4 \%$ to $84.1 \%$ in several studies worldwide. Non-steroid anti-inflammatory drugs (NSAID) are typically used as the primary treatment for this condition, but are limited by inadequate pain control, gastrointestinal discomfort and impact on renal function. Combined oral contraceptives are also frequently used but are not universally accepted perhaps due to its potential side effects on inducing endometriosis [2]. Acupuncture has gained more and more popularity as an alternative therapy in the past 40 years and been considered "essential health benefits" in the US and in high demand in many countries $[1,3]$. Not only Chinese herbal medicine (CHM), including crude herbs, is wellaccepted and commonly used in granule and powder in Taiwan to treat various health conditions, but acupuncture has gained more and more popularity worldwide with the efficacy of symptom relief given to women with primary dysmenorrhea [4].

\section{Differences of TCM and Western Medicine}

Reviews of literature about primary dysmenorrhea in both Western medicine, acupuncture, and traditional Chinese medicine are conducted on PubMed in this research. In this section, TCM perspective and Western medicine intervention are discussed.

TCM Perspective of Dysmenorrhea: The key principle of TCM is centered on the theory that harmony between Yin and Yang is the crux of health, whereas disharmony between Yin and Yang results in disease. Both Chinese herbs and acupuncture are two common modalities, which are based on the theory of Yin-Yang balance and the Five Element theory of TCM in clinic [1]. Blood (Xue) is considered to be the nourishing agent in TCM, which interacts with Qi, the moving agent to maintain the circulation in body [5]. Blood nourishes Qi and Qi moves Blood. One is inconceivable without the other, based on the concept in TCM that Qi is the commander of Blood, and Blood is the mother of Qi [6]. The circulation of Qi and Blood in the body should be constant, or the pain shall occur when the 
"free flow" of Qi and Blood is disrupted. The relationship therefore between Qi and Blood can be determined to be interpromoting, interdependent, and intertransforming [7]. The circulation of Qi and Blood depends on the correct, interdependent functioning of Zang-Fu organs, involving in Lung, Heart, Liver, Spleen, Kidney, and San Jiao as shown in Table 1. The Liver Qi keeps Qi circulate in the all parts of the body, connecting to the Spleen and promoting the digestion functions to produce Blood [6]. Mechanisms in the disorder of Qi and Blood include external factors like Wind and Heat, emotional disturbance, bad diet and physic trauma, and improper exertion in activities, such as sex or excessive study [8]. In addition to the discussion about the relation between Qi and Blood, one of the important functions of Blood in TCM is moistening and nourishing the skin, muscles, sinews, bones and internal organs, which is closely related to pain when Qi malfunctions.

Table 1: Functions of Zang organs related to Blood.

\begin{tabular}{|c|c|}
\hline Zang & Functions \\
\hline Liver & Storing Blood and regulating the volume of Blood \\
\hline Spleen & Controlling Blood \\
\hline Kidney & Controlling Bone \\
\hline
\end{tabular}

Pain, mainly a result of disturbances in the circulation of Qi and Blood, indicates the disharmony between Qi and Blood classified as stagnation of Qi and stasis of Blood, or Qi deficiency and Blood deficiency. Both Qi and Blood should circulate freely in the body, particularly in the Penetrating and Directing Vessels. There could be various causative factors leading to pain, but the main pathology is either due to blockage and obstruction that is Excess pattern, or to deficiency of Blood viewed as Deficiency pattern [6]. The key organ that causes of these issues is Heart because Heart can govern the blood and blood vessels and the root controller of Zang$\mathrm{Fu}$ organs [8]. External pathogenic factors, abnormal emotions, dysfunctions of internal Zang-Fu organs lead to dysmenorrhea. The pathogenic factors may cause either Qi stagnation or Blood stasis in the Penetrating and Direction Vessel to block the uterus or fail to regulate the Vessels or nourish poorly the uterus by Qi and Blood [6]. From the point of view of TCM, Liver, Penetrating and Directing Vessels are responsible for the physiology of menstruation. Proper movement of Blood relies mainly on the free flow of Liver Qi. In other words, Liver Qi and Liver Blood are essential for a pain-free period if they can move properly. Menstruation is identified from the four different phases as follows [9]:

Before Menstruation: Yang moves Blood, and Liver Qi moves in preparation to move Blood.

During Mid-Cycle: Blood and Yin fill up in the Penetrating and Directing Vessels.

During Menstruation: Blood moves with the help of Liver Qi and Liver Blood.
After Menstruation: Blood and Yin are empty.

\section{Treatments of Western Medicine for Pain in Dysmenorrhea}

In the pathological perspective of Western medicine, the pathogenesis of primary dysmenorrhea may have much to do with elevated levels of prostaglandins in response to the rise and fall of progesterone, which can occur after ovulation. Lysosomes in the endometrial cells break down and release phospholipase A 2, which converts cell membrane fatty acids into arachidonic acid as progesterone production decreases by the corpus luteum. The excessive elevation of prostaglandins leads to uterine hypercontractility, painful cramping and other nausea, vomiting, and diarrhea symptoms that are prostaglandin-related. Contractions caused by decreased blood flow to the uterus lead to ischemia, which makes nerve fibers sensitive to the inflammatory prostaglandins and endoperoxides1. On the other hand, the excessive level of hormone Vasopressin increases uterine contractility in the women with dysmenorrhea contributing to cramping and ischemia [10]. Current treatments in the Western medicine to menstrual pain are nutrition, supplements, botanicals, bioenergetics therapy, and pharmaceutical use. Omega-3 Fatty Acids is suggested because it may modulate the levels of prostaglandins, inflammation, and painful uterine contractions. The role magnesium plays in the treatment of menstrual pain lies in its effect on intracellular calcium concentration, leading to reduction in prostaglandin synthesis. This therapy can be given by foods like fish, nuts, leafy greens, whole grain cereals. In addition, Vitamin B2 (Pyridoxine) and Vitamin E are also suggested as supplements. In botanicals, French Maritime pine bark extract, Fennel and SCA (Saffron, Celery seed, Anise or Fennel) are given to the patients for the treatments.

Heat therapy is also reported to be beneficial to pain relief, particularly with NSAIDs, such as ibuprofen, ketoprofen, mefenamic acid, naproxen, and cyclooxygenase (COX) -2 inhibitor celecoxib3. Laparoscopy is performed when conservative pharmacological management has failed. Pharmaceuticals used for relieving pain include nonsteroidal anti-inflammatory drugs (NSAIDs), such as naproxen, ibuprofen, and mefenamic acid. Cyclooxygenase (COX)2 inhibitor is considered the first-line reactive treatment with the use of hormonal modulation; for example, oral contraceptive pills. However, NSAIDSs can be associated with significant side effects, causing gastrointestinal symptoms like nausea and indigestion. It is also pointed out that NSAIDs are associated with higher risk of headache, dizziness, drowsiness, and dry mouth than placebo [11].

The result of relieving pain of a study shows that acupuncture therapy of the TCM can relieve pain in the patients with dysmenorrhea, promoting Qi and Blood circulation [12]. Whether or not the efficacy of acupuncture is better than other interventions is also confirmed with the conclusion that "Acupuncture was as effective as NSAID therapy for patients with primary dysmenorrhea." 
In this study, Group 1 received NSAIDs and group 2 received acupuncture care. The result shows that one month later the NSAID group showed a $52.2 \%$ decrease in dysmenorrhea related pain, but the acupuncture group demonstrated a $69.5 \%$ decrease in dysmenorrhea related pain [13]. In terms of the use of TCM herbs for dysmenorrhea, pattern identification plays a key role which can affect the treatments. It is presented in Table 2 that the most common patterns in Taiwan are Liver blood deficiency, Liver qi stagnation, and Blood stasis, highlighting the importance of Liver in this health issue [14]. In the TCM theories, Liver Qi is the key to the treatment of dysmenorrhea with the functions of regulating Blood and dispersing stagnated Qi. However, Liver Qi is easily stagnated with the emotional stimulation like anger, frustration, resentment, stress, etc. In addition to Liver, causative factors are Qi and Blood Deficiency, and Liver and Kidney Deficiency of Essence [5]. The general principle of treatment of dysmenorrhea is to regulate Qi and Blood in the Penetrating and Directing Vessels. Acupoints suggested for the acupuncture treatment are shown in Table 3.

Table 2: Frequently used prescriptions for PM.

\begin{tabular}{|c|c|c|}
\hline Prescriptions & Constituents & TCM patterns \\
\hline Dang-Gui-Shao-Yao San & $\begin{array}{c}\text { Angelicae sinensis, Ligustici chuanxiong, Paeoniae alba, Atractylodis } \\
\text { macrocephalae, Poriae cocos, and Alisma plantago-aquatica }\end{array}$ & $\begin{array}{l}\text { Liver blood deficiency } \\
\text { Liver-Spleen disharmony }\end{array}$ \\
\hline Jia-Wei-Xiao-Yao-San & $\begin{array}{l}\text { Paeoniae alba, Bupleurum chinense, Atractylodis macrocephalae, Poriae cocos, } \\
\text { Angelicae sinensis, Mentha haplocalyx, Glycyrrhizae uralensis, Zingiberis } \\
\text { officinalis recens, Paeonia suffruticosa radicis, and Gardeniae jasminoidis }\end{array}$ & $\begin{array}{l}\text { Liver qi stagnation and Spleen } \\
\text { deficiency }\end{array}$ \\
\hline Wen-Jing-Tang & $\begin{array}{c}\text { Evodia rutaecarpa, Cinnamomum cassia, Angelica sinensis, Paeoniae alba, } \\
\text { Ligustici chuanxiong, Panax ginseng, Glycyrrhizae uralensis, Equus asinus, } \\
\text { Ophiopogon japonicus, Pinellia ternata, and Zingiberis recens }\end{array}$ & Deficient cold and blood Stasis \\
\hline Gui-Zhi-Fu-Ling-Wan & $\begin{array}{c}\text { Cinnamomum cassia, Poriae cocos, Paeoniae alba, Paeonia suffruticosa, and } \\
\text { Prunus persica }\end{array}$ & Blood stasis in uterus \\
\hline Shao-Fu-Zhu-Yu-Tang & $\begin{array}{c}\text { Foeniculum vulgare, Zingiberis recens, Cinnamomum cassia Angelicae } \\
\text { sinensis, Ligustici chuanxiong, Peoniae rubra, Trogopterus xanthipes, Typha } \\
\text { Angustifolia, Myrrha, Corydalis yanhusuo }\end{array}$ & Qi stagnation, Blood stasis \\
\hline
\end{tabular}

Table 3: Acupoints prescription suggested for PM.

\begin{tabular}{|c|c|c|}
\hline Patterns & Acupoints & Indications \& Functions \\
\hline \multirow{3}{*}{ Liver Qi Stagnation } & PC-6 & To unblock the chest and calm the Mind. \\
\hline & LI-4 \& LV-3 & Viewed as "the four gate points" to promote the circulation of Qi and Blood \\
\hline & LV-2 & to regulate Liver Blood with fire \\
\hline \multirow{3}{*}{ Qi and Blood Deficiency } & $\mathrm{CV}-12 \& \mathrm{KD}-3$ & $\begin{array}{l}\text { To tonify Kidney and Original Qi to benefit the Essence and reinforce the } \\
\text { Blood. }\end{array}$ \\
\hline & ST-36 \& SP-9 & To activate Spleen and Stomach to reinforce the Blood. \\
\hline & SP-6 & To activate the Spleen and tonify the Blood. \\
\hline \multirow{4}{*}{ Liver and Kidney Deficiency of Essence } & KD-3 \& CV-6 & To tonify the Kidney to reinforce KD Qi and regulate menstruation. \\
\hline & SP-6 & To tonify three Yin channels of the foot. \\
\hline & KD-6 \& KD-7 & To nourish the Yin and clear Deficient-Heat. \\
\hline & KD-10 & To regulate the menstruation and clears Deficient-Heat. \\
\hline
\end{tabular}

\section{Discussion}

The Zang (臟)-Fu (腑) theory, the Five Elements theory and patterns are unique and absolutely different from those theories of the Western medicine. In general, those who have not learned TCM are usually confused with the functions of internal organs (ZangFu) in the TCM. TCM doctors use a special well-organized diagnosis system and syndrome differentiation called pattern identification for treatment, absolutely different from disease diagnosis in the western medicine, It has been reported that TCM herbs are superior to Western medications in treatment of dysmenorrhea in some aspects, such as lowering the recurrence rate and constitution adjustment, which includes modifying mood or correcting luteal insufficiency. To adjust one's constitution and relieve symptoms by administering a single prescription, such as mood modifying and analgesia in primary dysmenorrhea [14]. Acute pain can be mostly driven by central mechanisms while persistent pain is also largely driven by peripheral mechanisms. It is also suggested that there could not specific pathological factors in both primary and secondary dysmenorrhea. However, it is assumed contractions of the uterus that expel menstrual uterine constituents produce high-intensity stimuli. The sensitization or activation of uterine afferent neurons could be produced by the production of various 
products of inflammation by the sloughing necrotic uterine lining [11]. On the other hand, recent developments in neuroscience have enriched the understanding of pain drive, showing there could be more than one pain drivers in one patient. Multiple mechanisms need healthcare providers' attentions when facing patients with pain [12]. It is presented that prolonged or habitual emotional disturbance can lead to Liver Qi stagnation in the Penetrating and Directing Vessels, and the Uterus.

From the perspective of Traditional Chinese Medicine, obstructions of Qi and Blood can block the channel, causing pain during menstruation with inflammation [9]. Sanyinjiao (SP6, 三 (san)-陰(Yin)-交(jiao)) in this prescription, first discussed in Zhen Jiu Jia Yi Jing (The Systematic Classic of Acupuncture and Moxibustion, 針炎甲乙經 ), is on the Spleen meridian of Foot Taiyin, and is the meeting point of three Yin meridians: Spleen, Liver, and Kidney. This Foot Taiyin meridian intersects the Conception vessel, enters the Spleen, connects with the Stomach, links with the Heart, passes through LV 14, and finally spreads over the lower surface of the tongue. This acupoint included in The Song of the nine needles for returning Yang (回陽九針歌) is usually used to benefit the Spleen and Kidney, transform dampness, and spread the stagnated Liver qi. SP6 is commonly used, based on the actions such as the tonification of Spleen and Stomach through resolving dampness, harmonization of Liver, regulation of urination and menstruation by invigorating Blood [6]. Kidney in the TCM is referred to as the "Root of Life" because it stores Essence (Jing, 精). Essence is the creator of life and the foundation for Yin and Yang. On the other hand, the essence the Kidney stores is the foundation of producing Marrow, which is often misunderstood because it cannot exactly correspond to bone marrow in the Western medicine. Essence can be transformed into Blood, and Blood nourishes Essence. In other words, both Blood and Essence interact with each other to maintain the Yin level for normal functions in the body [6]. The most important reason why SP6 is frequently used for obstetric and gynecologic issues lies in Blood and for the actions to [6]:

a) Tonify the Spleen and the Stomach

b) Eliminate Dampness

c) Nourish the Blood and Yin

d) Regulate menstruation

e) Promote labor

f) Regulate urination

g) Benefit the genitals

h) Harmonize the Lower Burner

i) Calm the Shen (神)

Blood circulation benefits the tissues to fight with inflammation.
Inflammation, to a certain extent, is viewed as obstruction which can gradually cause Qi in Liver and Kidney and Blood Deficiency from the aspect of the TCM [6]. It is confirmed and agreed the stimulation at the acupoints with acupuncture could increase blood flow velocity of the peripheral arterioles and oxygenated hemoglobin to modulate the blood circulation of the body surface. As blood circulation increased in the affected areas, proinflammatory response decreased with regulation of inflammation process and growth factors $[15,16]$. The role of acupuncture in stimulating immune function, including increasing blood cell count containing hemoglobin and enhancing lymphocyte and natural killer cell activity, has been confirmed [16].

Hemoglobin in blood cell binds to either oxygen or carbon dioxide. This binding allows oxygen to be transported around our body to our tissues and organs, and carbon dioxide to be taken away. However, the number of hemoglobin relies on the functions of Kidney in the TCM and the Western medicine [16]. The literature of oxygen metabolism, the theory of meridians, and Qi in the meridians highlights that both Qi and oxygen have informational, material, and functional features, and bear high similarity in physiological functions and pathological reactions [17]. One of the functions of the kidney is to set the hematocrit at a normal value of $45 \%$ to maximize oxygen delivery [18]. The rising formation of red blood cells, which are promoted by the hormone Erythropoietin (EPO) produced by the kidney, increases the oxygen-carrying capacity of the blood. However, it is stated that the production of Erythropoietin is determined by tissue oxygen pressure. In other words, the kidney is closely connected to the oxygen circulation $[18,19]$. This statement highlights the functions of Kidney in the TCM. Kidney qi, to some extent, can be viewed as blood oxygen [1]. Qi, a vague term, is one of the important concepts in the TCM that cannot be defined well and exactly in the Western medicine, but it can nourish the tissues and help digestion and absorption to produce Essence in the theory of the TCM as long as Qi can flow freely $[20,21]$. Blood circulation increases when Qi is circulating within the body smoothly, which is verified by the conclusion of a study that the blood circulation level increased with acupuncture treatment, which corresponds to the function of Qi in the TCM that Qi can move Blood to relieve the pain as shown in Figure $1[17,18]$. In terms of gynecologic complaints, including dysmenorrhea, Dong quai is the most popular herb with Chinese and has been used commonly in TCM formulas with the TCM diagnoses of "blood stasis" and "blood vacuity," associated with the diagnoses of amenorrhea, dysmenorrhea, uterine fibroids, and endometriosis in Western medicine. However, interaction risks of Chinese herbs, including Dong quai, and Western medications deserve attention as in Table 4 [6]. In addition to interactions, side effects can also arise from the uses of Western medications. Dampness, which can be caused by Antihistamine and Steroid, is one of the Six Evils in TCM that can induce Blood deficiency and Qi stagnation. 


\section{Input Dysmenorrhea \\ Output \\ Blood \& Oxygen \\ Endogenous opioid \\ Prostaglandin $\downarrow$. \\ M1 Macrophage $\downarrow$. \\ $\uparrow \mathrm{M} 2$ Macrophage. \\ Pain relieved}

Figure 1: The functions of Qi and Blood in pain relief.

Table 4: Interactions of Dong Quai (Radix Angelicae Sinensis) with Western medications.

\begin{tabular}{|c|c|c|}
\hline Western medications & Interaction Rating & Risks \\
\hline Warfarin (Coumadin) & Major & To increase the chances of bruising and bleeding. \\
\hline naproxen, ibuprofen, and mefenamic acid & Moderate & To increase the chances of bruising and bleeding. \\
\hline Estrogens & Moderate & To increase the risk for side effects with acting like the hormone estrogen \\
\hline
\end{tabular}

\section{Conclusion}

Discussions suggest that acupuncture can control or reach the goal of pain relief for dysmenorrhea as Western medicine. However, integration of Western medicine and TCM for treating dysmenorrhea needs the further consideration of side effects it can cause. Recent researches on this issue have not been presented, and it implies the integration deserves further work. TCM pattern prevalence in Taiwan may be different from that in the western country with geographical factors like diet and weather, which should be taken into consideration for treatment. Along with the use of NSAIDs, which are associated with side effects of headache, dizziness, drowsiness, and dry mouth, feasible selection of acupoints and needling manipulations are required.

\section{References}

1. Hong TZ (2017) Exploring a new extra point for sub-acute cough: A case report. Scholar's Press, Germany.

2. Behbahani BM, Ansaripour L, Akbarzadeh M, Zare N, Hadianfard MJ (2016) Comparison of the effects of acupressure and self-care behaviors training on the intensity of primary dysmenorrhea based on McGill pain questionnaire among Shiraz University students. J Res Med Sci 21: 104.

3. Armour M, Dahlen HG, Zhu X, Farquhar C, Smith CA (2017) The role of treatment timing and mode of stimulation in the treatment of primary dysmenorrhea with acupuncture: An exploratory randomised controlled trial. PLoS One 12 (7): e0180177.

4. Pan J, Tsai Y, Lai J, Fang R, Yeh C (2014) The traditional Chinese medicine prescription pattern of patients with primary dysmenorrhea in Taiwan: A large-scale cross sectional survey. J Ethnopharmacol 152(2): 314-319.

5. Chu Q, Gong C (2014) Acupuncture for dysmenorrhea, Internatioal Journal of Clinical Acupuncture 23(2): 67-73.

6. McPHEE SJ, Papadakis MA (2012) Current Medical Diagnosis \& Treatment.

7. Hong TZ (2017) Acupressure or Acupuncture at Sanyinjiao (SP6) for Primary Dysmenorrhea. J Network Med Target Ther 1(1).
8. Sun P (2011) Treatment of pain with Chinese herbs and acupuncture. Churchill Livingstone.

9. Zhu B, Wang H (2010) Basic theories of traditional Chinese medicine. People's Military Medical Press. PA, USA.

10. Maciocia G (1994) The practice of Chinese medicine: The treatment of diseases with acupuncture and Chinese herbs. Churchill Livingstone.

11. Rakel D (2012) Integrative Medicine.

12. McMahon SB, Koltzengburg, Wall, Melzack's Textbook of Pain, Churchill Livingstone.

13. Chaitow L, Jones RL (2006) Chronic pelvic pain and dysfunctionpractical physical medicine. Churchill Livingstone.

14. Kiran GS, Gumusalan Y, Ekerbiçer HC, Kiran H, Coşkun A, et al. (2013) A randomized pilot study of acupuncture treatment for primary dysmenorrhea. Eur J Obstet Gynecol Reprod Biol 169(2): 292-295.

15. Chen H, Lin Y, Su IH, Chen Y, Yang S, et al. (2014) Investigation on Chinese herbal medicine for primary dysmenorrhea: implication from a nationwide prescription database in Taiwan. Complement Ther Med 22(1): $116-125$.

16. Litscher G (2006) Bioengineering assessment of acupuncture, part 2: Monitoring of microcirculation. Crit Rev Biomed Eng 34(4): 273-294.

17. Hsiu H, Hsu WC, Chen BH, Hsu CL (2010) Differences in the microcirculatory effects of local skin surface contact pressure stimulation between acupoints and nonacupoints: possible relevance to acupressure. Physiol Meas 31(6): 829-841.

18. Xu BQ (1986) Experimental studies on acupuncture treatment of acute bacillary dysentery-The role of humoral immune mechanism. In Zhang XT (Eds.). Researches on acupuncture-moxibustion and acupunctureanesthesia. Science Press, pp. 573-578.

19. Donnelly S (2001) Why is erythropoietin made in the kidney? The kidney functions as a critmeter. Am J Kidney Dis. 38(2): 415-425.

20. Nabili SN Erythropoietin (EPO, The EPO Test).

21. Kubo K, Yajima H, Takayama M, Ikebukuro T, Mizoguchi H, et al. (2011) Changes in blood circulation of the contralateral Achilles tendon during and after acupuncture and heating. Int J Sports Med 32(10): 807-813. 
This work is licensed under Creative Commons Attribution 4.0 License

To Submit Your Article Click Here: Submit Article

DOI: 10.32474/OAJCAM.2018.01.000111

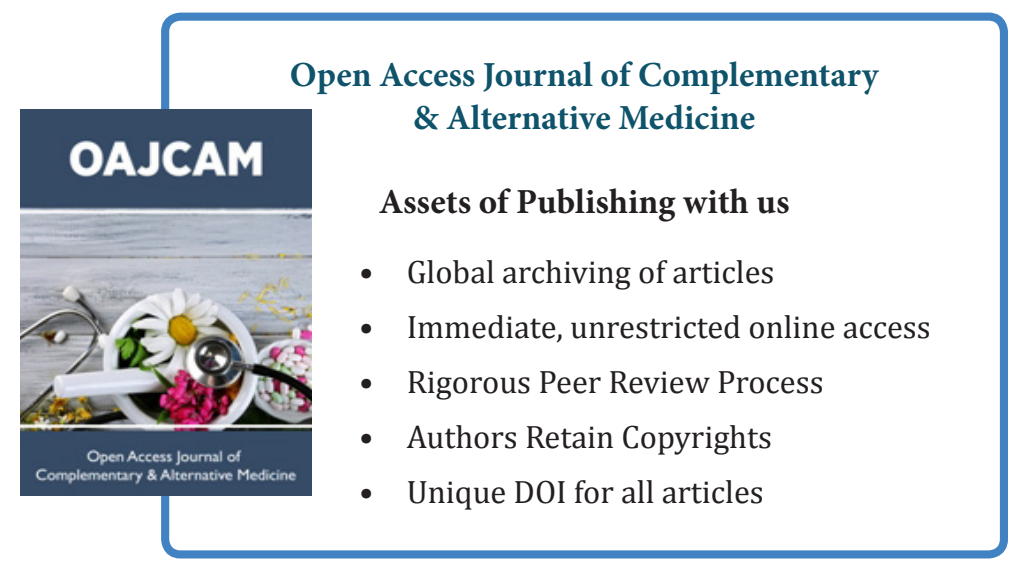

